\title{
Design of a neuroastrocytic model with dopaminergic NT modulated by astrocytes in the Prefrontal Cortex
}

\section{Sara Alvarez-Gonzalez, Francisco Cedron}

In this project, we show a new neuroscientific approach to information transmission and modulation in which networks of neurons and astrocytes mediate, thanks to the action of which, the processing of the received information is improved. The prefrontal cortex is in charge of cognitive control, and the information that arrives is processed from the beginning in that area to make decisions based on it. With the elapse of seconds after the information is processed by the brain, certain neuromodulators, such as dopamine in the hippocampus, are released and connect with the prefrontal cortex to modulateattention,impulse inhibition, memory or cognitive flexibility from how it was being done up to that moment.It is from this idea that a new computational model, composed of a Deep Learning network (representing the prefrontal cortex), a network of artificial neurons (representing the dopaminergic network) and the presence of an intermediate mediating component which would be the astrocyte, will be carried out. Thisnew computational system shows improvements in the resolution of the AA problems it has faced, from the model with the DL network working inisolation, and even, improvement of the networks working together is observed if the astrocyte is in operation. Improvement also observed in biological brain processing. 